\title{
A Current-Commutation DC Circuit Breaker with Adaptive Reclosing Capability
}

\author{
Chunbing Jiang ${ }^{1}$, Chengyong Zhao ${ }^{1 *}$ \\ ${ }^{1}$ State Key Laboratory of Alternate Electrical Power System with Renewable Energy Sources, North China Electric Power University, \\ Beijing, 102206, China
}

\begin{abstract}
DC faults are critical events in a flexible high-voltage dc (HVDC) grid. Thus, ensuring that the power system returns to normal operation rapidly and reliably after fault isolation is very important. This requires a HVDC breaker. In overhead line systems under temporary faults, reclosing is often required. However, once the dc circuit breaker (DCCB) is reclosed directly, the large second overcurrent may occur which could damage the power electronic devices. To avoid this problem, a current-commutation DC circuit breaker with adaptive reclosing capability is proposed. Compared with the traditional auto-reclosing strategy, the second damage under permanent fault condition can be avoided by the proposed DCCB, which can identify the fault property. Compared with the hybrid DCCB, the power electronic breaking branch composed of lots of IGBTs is replaced by the current- commutation branch, which is employed to interrupt bi-directional dc fault current. Moreover, bypassing branch is configured to reduce the energy dissipation of arrester and shorten the fault isolation time effectively. Finally, simulation cases in PSCAD /EMTDC verify the effectiveness and superiorities of the proposed DCCB.
\end{abstract}

\section{Introduction}

The voltage source converters based HVDC grids are expected to integrate large-scale renewable energy located far from the load center in the future [1]-[2]. Especially, with the development of the modular multilevel converter (MMC) [3], it becomes more feasible to apply the flexible HVDC transmission.

The overhead line will be widely applied in the future HVDC grids because of the economic advantage. Compared with the dc cable, the temporary fault probability of the overhead line is much higher. Hence, effective reclosing strategies should be applied to improve the HVDC system reliability. Moreover, the dc line impedance is much smaller than the one in the ac system [4], causing the dc fault current to rise rapidly within a few milliseconds. Therefore, one of the major challenges of HVDC grids is the fast and reliable isolation of dc faults [5]. The hybrid DCCB is an effective solution to interrupt dc fault current quickly [6]. However, the power electronic breaking branch composed of lots of IGBTs and diodes needs to withstand dc system voltage. Thus, the hybrid DCCB is composed of a large number of power electronic devices, leading to a high cost of hybrid DCCB. The reduction of the peak fault current will reduce the energy dissipation and the capital cost of DCCB. Once the fault is isolated, the key problem for the reclosing is to identify the fault property (temporary or permanent) reliably. Generally, the second damage under permanent fault condition in the flexible HVDC grid is much more severe, while the power electronic devices are very sensitive. Thus, it is not appropriate to reclose the DCCB directly for recovering the system anymore. Above all, the low-cost DCCB which can identify the fault property still needs further research.

Reference [7] designed a low-cost voltage clamping based DCCB, but it can only interrupt the current in one direction. Reference [8] proposed a DCCB with H-bridge circuit composed of diode groups to achieve the ability of bi-directional current breaking. Compared with the hybrid DCCB proposed by ABB, the number of IGBTs is halved, but the fault current is not suppressed significantly. Reference [9] proposed a DCCB with Hbridge circuit, which the fault current is suppressed dramatically. However, there are still a large number of IGBTs in the power electronic breaking branch. Besides, it is worth to mention that permanent faults and temporary faults cannot be identified in this scheme, which has the risk to reclose under permanent fault condition directly, so that the large second overcurrent may occur to damage the electronic devices in the grid. In order to further reduce the cost of DCCB and avoid reclosing under permanent fault condition directly, a current-commutation DC circuit breaker with adaptive reclosing capability is proposed. 


\section{A current-commutation DC circuit breaker with adaptive reclosing capability}

Topology of the proposed DCCB is shown in Figure. 1, which is composed of the current-flow, current commutation, bypassing and reclosing identification branches. In addition, the current-limiting inductor of the dc line is represented as $L_{\mathrm{dc}}$.

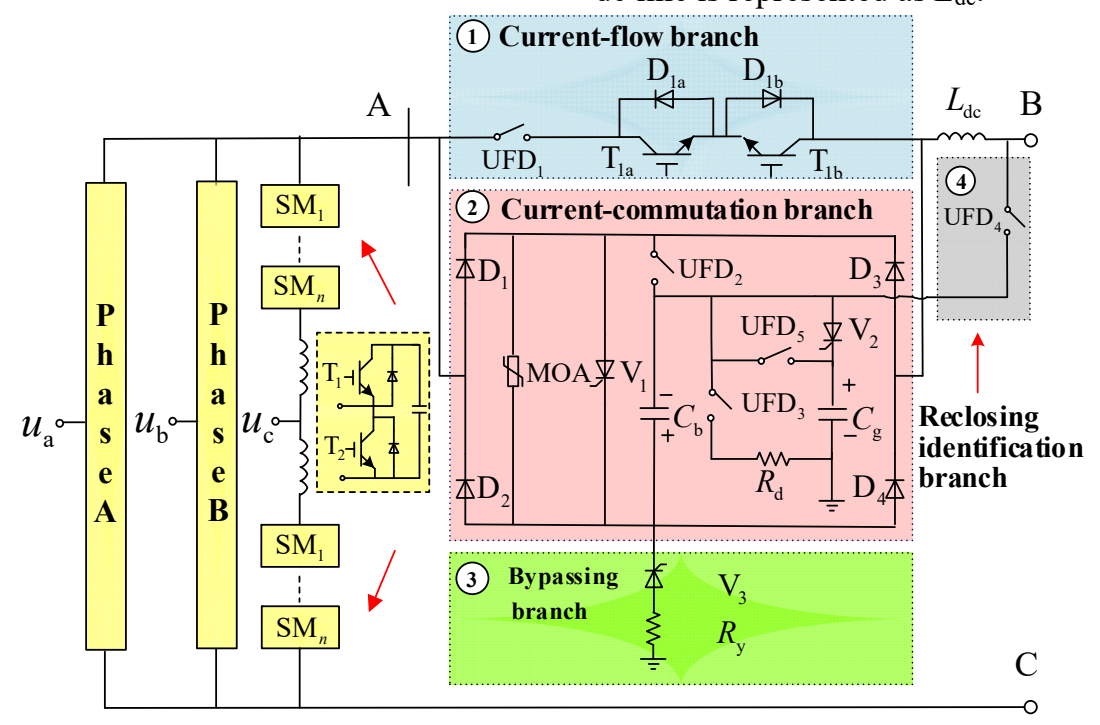

Figure 1. Topology of the current-commutation DC circuit breaker with adaptive reclosing capability

\section{Stage 1: $\underline{t}_{0} \underline{\underline{\leq}} \underline{\underline{t_{1}}} \underline{1}_{1}$}

The fault is assumed occurring at $t_{0}$. Considering the delay of fault detection, the fault has been detected and located at $t_{1}, \mathrm{~T}_{1 \mathrm{a}}$ and $\mathrm{T}_{1 \mathrm{~b}}$ will be switched off immediately to commutate the fault current from current-flow branch to the current-commutation branch, and then $\mathrm{UFD}_{1}$ is subsequently opened.

\section{Stage 2: $t_{1}<t<t_{2}$}

At $t_{1}, \mathrm{~V}_{1}$ and $\mathrm{V}_{2}$ will be triggered, ensuring the reliable opening of $\mathrm{UFD}_{1}$ and LCS at zero voltage and zero current. At the same time, $\mathrm{UFD}_{1}$ will be switched off and $\mathrm{UFD}_{2}$ will be switched on. $C_{\mathrm{g}}$ is used to regulate the charging voltage of $C_{\mathrm{b}}, C_{\mathrm{b}}$ will be charged to a negative voltage for the reliable turning-off of $V_{1}$ in the next stage. When the charging process is finished, $\mathrm{V}_{2}$ will turn off automatically.

Stage 3: $t_{2}<t<t_{3}$

At $t_{2}$, the contactor of the $\mathrm{UFD}_{1}$ has separated completely and the contactor of the $\mathrm{UFD}_{2}$ has connected completely. At the same time, $\mathrm{V}_{1}$ will turn off after a few milliseconds because of the negative voltage of the commutation capacitor $C_{\mathrm{b}}$, and the current starts to commute from $\mathrm{V}_{1}$ branch to $\mathrm{UFD}_{2}$ branch. With the reverse charging of $C_{\mathrm{b}}$, the voltage of $C_{\mathrm{b}}$ increases gradually. When the capacitor voltage equals to the voltage of HVDC system, the current of $C_{\mathrm{b}}$ starts to decrease, and the voltages on $L_{\mathrm{dc}}$ and $L_{\mathrm{L}}$ become negative. With the increase of time, the voltage exerted on $C_{\mathrm{b}}$ becomes higher than the voltage of the HVDC system.

\section{Stage 4: $\underline{t}_{3}<t<t_{4}$}

At $t_{3}$, when the voltage exerted on $C_{\mathrm{b}}$ reaches the triggering threshold of MOA, the fault current starts to commute to dissipation branch as shown by $i_{5}$ in Figure. 2(d). Meanwhile, $V_{3}$ will be triggered to bypass the line inductor $L_{\mathrm{dc}}$, so that the energy dissipation of MOA is reduced and the fault clearing time is shortened. When the fault current of bypassing branch turns to be zero, $V_{3}$ will automatically turn off. At $t_{4}$, the fault current will drop to zero, which means DC fault has been isolated successfully. At the same time, $\mathrm{UFD}_{2}$ will start to be switched off, further preparing for reclosing identification.

\section{Stage 5: $t_{5} \leq t<t_{6}$}

At $t_{5}, \mathrm{UFD}_{3}, \mathrm{UFD}_{4}$ will be turned on to drain the energy of $C_{\mathrm{b}}$ and identify the fault property. At $t_{6}$, the contactors of $\mathrm{UFD}_{3}$ and $\mathrm{UFD}_{4}$ have connected completely, and the gap between $t_{5}$ and $t_{6}$ is $2 \mathrm{~ms}$, and $\mathrm{V}_{3}$ will be trigged at this moment. Considering that electromagnetic induction phenomena cannot be ignored in large-scale DC grids, the current of reclosing identification branch is difficult to turn to zero approximately even the temporary fault has eliminated. Hence, the identification threshold value $I_{\text {th }}$ is introduced to avoid misjudgment, which can be set to $2 \mathrm{kA}$. When the fault is temporary fault, the fault point does not exist at $t_{5}$. Thus, the current of reclosing identification branch is less than $I_{\text {th }}$. When the fault is a permanent fault, the fault point still exists at $t_{5}$, the discharging branch and the reclosing identification branch both can provide discharging paths for $C_{\mathrm{b}}$. The current of reclosing identification branch is greater than the identification threshold value $I_{\text {th }}$. When the discharging process is finished, $V_{3}$ will automatically turn off. When the current of $\mathrm{UFD}_{4}$ turns to be zero, the $\mathrm{UFD}_{4}$ can be switched off. Obviously, the fault is a temporary fault or a permanent fault can be identified according to the current of reclosing identification branch.

\section{Stage 6: $t_{7}<t<t_{9}$}

At $t_{7}, \mathrm{UFD}_{5}$ will be switched on to drain the energy of the grounded capacitor $C_{\mathrm{g}}$. At $t_{8}$, the contactor of $\mathrm{UFD}_{5}$, 
have connected completely, the gap between $t_{7}$ and $t_{8}$ is $2 \mathrm{~ms}$. At $t_{9}$, when the discharging process is finished, the $\mathrm{UFD}_{3}$ and $\mathrm{UFD}_{5}$ will start to open. In order to ensure the HVDC system to return to normal operation reliably after fault isolation, the $\mathrm{UFD}_{1}$ and $\mathrm{UFD}_{2}$ should be switched on firstly, and then the $T_{1 a}$ and $T_{1 b}$ will be triggered. During the dc steady state, the current-commutation branch, bypassing branch and reclosing identification branch can be considered as open circuits.

\section{Parameter analysis}

\subsection{Design of the Capacitance}

The commutation capacitor $C_{\mathrm{b}}$ is required to be charged with reverse voltage during stage $2 \mathrm{I}$, so that $\mathrm{V}_{1}$ can withstand reverse voltage and turn off properly at $t_{2}$. The charging of the commutation capacitor $C_{\mathrm{b}}$ and the grounded capacitor $C_{\mathrm{g}}$ depends on the voltage of dc line $U_{\mathrm{dc}}$. The charging path is as shown by $i_{3}$ in Figure. 2(b). During $t_{2} \sim t_{3}$, the charging path of the $C_{\mathrm{b}}$ is as shown by $i_{4}$ in Figure. 2(c). The fault current of $V_{1}$ branch is considered to be commuted to $\mathrm{UFD}_{2}$ branch immediately at $t_{2}$. The initial conditions are $i_{\mathrm{d}}\left(t_{2}\right)=i_{\mathrm{dc}}\left(t_{2}\right)=I_{1}, U_{\mathrm{d}}\left(t_{2}\right)=$ $U_{\mathrm{dc}}\left(t_{2}\right)=U_{1}$, ignoring the time that the fault current of $\mathrm{V}_{1}$ drops to zero. The current of $C_{\mathrm{b}}$ in time domain can be described as:

$$
i_{\mathrm{d} 1}(t)=\frac{e^{-\omega_{\mathrm{S} S t} t}}{\sqrt{1-\delta_{2}^{2}}}\left[\frac{U_{1} C_{\mathrm{M}} C_{\mathrm{b}} \omega_{1}}{\left(C_{\mathrm{M}}+C_{\mathrm{b}}\right)} \sin \left(\omega_{1} \sqrt{1-\varsigma_{1}^{2}} t\right)-I_{1} \sin \left(\omega_{1} \sqrt{1-\varsigma_{1}^{2}} t-\gamma_{1}\right)\right]
$$

Based on equation (1), the voltage of the commutation capacitor $C_{\mathrm{b}}$ in time domain can be expressed as:

$$
U_{\mathrm{Cb}}(t)=\frac{1}{C_{\mathrm{b}}} \int_{t 2}^{t} i_{\mathrm{d} 1}(t) \mathrm{d} t \quad t_{2}<t<t_{3}
$$

According to equation (2), with the value of $C_{b}$ decreasing, the charging speed of $C_{\mathrm{b}}$ increases. At $t_{3}$, the voltage exerted on $C_{\mathrm{b}}$ reaches the triggering threshold of MOA, the current starts to commute from $C_{\mathrm{b}}$ branch to dissipation branch. The triggering time of MOA is usually taken as the turn-off time in traditional hybrid DCCB, which is $5 \mathrm{~ms}$ properly. According to equation (5), if the turn-off time is $5 \mathrm{~ms}$, the upper limit value $C_{\mathrm{b}_{-} \max }$ of $C_{\mathrm{b}}$ can be calculated as $30 \mathrm{uf}$.

\subsection{Design of the bypassing resistor $R_{y}$}

As the previous section mentioned, the bypassing thyristor $V_{3}$ will be turned on in stage 4 and stage 5 . The design of the bypassing resistor $R_{\mathrm{y}}$ is related to the current of the bypassing branch. The currents of bypassing branch in stage 4 and stage 5 need to be discussed, respectively.

In stage $4, V_{3}$ will be triggered to bypass the line inductance $L_{\mathrm{dc}}$ at $t_{3}$. The initial conditions are $i_{\mathrm{d}}\left(t_{3}\right)=$
$i_{\mathrm{dc}}\left(t_{3}\right)=I_{2}$. The current of the bypassing branch in stage 4 in time domain can be given as:

$$
i_{\mathrm{d} 2}(t)=I_{2} e^{-\left(R_{\mathrm{y}}+R_{\mathrm{L}}\right) t /\left(L_{\mathrm{dc}}+L_{\mathrm{L}}\right)}
$$

In stage 5, when the fault is a permanent fault, the fault point still exists at $t_{5}$. The discharging branch and the reclosing identification branch both can provide discharging paths for $C_{\mathrm{b}}$. The current of the bypassing branch decays exponentially. The initial conditions are $i_{\mathrm{d}}\left(t_{5}\right)=i_{\mathrm{Cb}}\left(t_{5}\right)=I_{3}, U_{\mathrm{d}}\left(t_{5}\right)=U_{\mathrm{Cb}}\left(t_{5}\right)=U_{3}$. The current of the bypassing branch in stage 5 in time domain can be expressed as:

$$
i_{\mathrm{d} 3}(t)=\frac{e^{-\omega_{2} \zeta_{2} t}}{\sqrt{1-\varsigma_{2}^{2}}}\left[U_{3} C_{\mathrm{b}} \omega_{2} \sin \left(\omega_{2} \sqrt{1-\varsigma_{2}^{2}} t\right)-I_{3} \sin \left(\omega_{2} \sqrt{1-\varsigma_{2}^{2}} t-\gamma_{2}\right)\right]
$$

According to equation (3) and equation (4), the maximum currents of the bypassing branch represented as $i_{\mathrm{d} 2 \text { max }}$ and $i_{\mathrm{d} 3 \_ \text {max }}$ can be calculated, respectively. In view of the current limit of the bypassing thyristor $V_{3}$, the maximum currents of the bypassing branch should be limited to no more than the overcurrent limit of $\mathrm{V}_{3}$. The bypassing resistor $R_{\mathrm{y}}$ can be taken as $100 \Omega$.

\section{Case study}

In order to verify the effectiveness of the proposed DCCB, a four-terminal dc grid model is built in PSCAD/EMTDC, whose topology is shown in Figure.2.

\subsection{Fault isolation process}

The pole-to-ground fault occurs at $t_{0}=1.0 \mathrm{~s}$, located at the head of line 1_4 as Figure. 3 shows. The fault isolation process of the proposed DCCB is shown in Figure.6. As shown in Figure. 3(a), the current of current-flow branch increases rapidly once the fault occurs. Considering the operation delay caused by fault detection and location, $\mathrm{V}_{1}$ is triggered at $1.001 \mathrm{~s}$. Meanwhile, the $\mathrm{T}_{1 \mathrm{a}}$ and $\mathrm{T}_{1 \mathrm{~b}}$ are turned off, so that the current of current-flow branch has turned to be zero, which provides $\mathrm{UFD}_{1}$ with zero current turn-off condition. At $1.003 \mathrm{~s}$, the contactors of the UFD have separated completely and the contactors of the $\mathrm{UFD}_{2}$ have connected completely, so that the fault current starts to commute from $\mathrm{V}_{1}$ branch to $\mathrm{UFD}_{2}$ branch. At $1.005 \mathrm{~s}$, the voltage exerted on $C_{\mathrm{b}}$ reaches $U_{\text {trigger}}$, and the fault current starts to commute from $C_{\mathrm{b}}$ branch to dissipation branch. At the same time, $V_{3}$ will be triggered to bypass the current-limiting inductor $L_{\mathrm{dc}}$. When the current of bypassing branch turns to be zero, $\mathrm{V}_{3}$ will automatically turn off. As shown in Figure. 3(b), $C_{\mathrm{b}}$ and $C_{\mathrm{g}}$ are charged by dc line at $1.001 \mathrm{~s}$. At $1.003 \mathrm{~s}, C_{\mathrm{b}}$ is charged reversely due to the commutation of the fault current. Differently from $C_{\mathrm{b}}, C_{\mathrm{g}}$ will not be charged anymore because $\mathrm{V}_{2}$ has already turned off before $1.003 \mathrm{~s}$. 


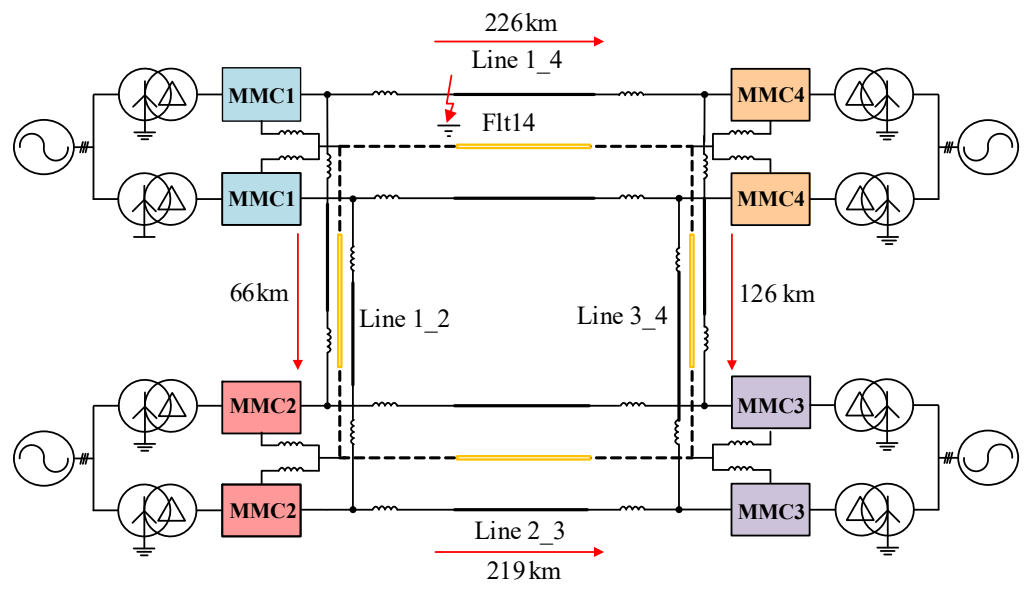

Figure 2. Zhangbei four-terminal HVDC grid
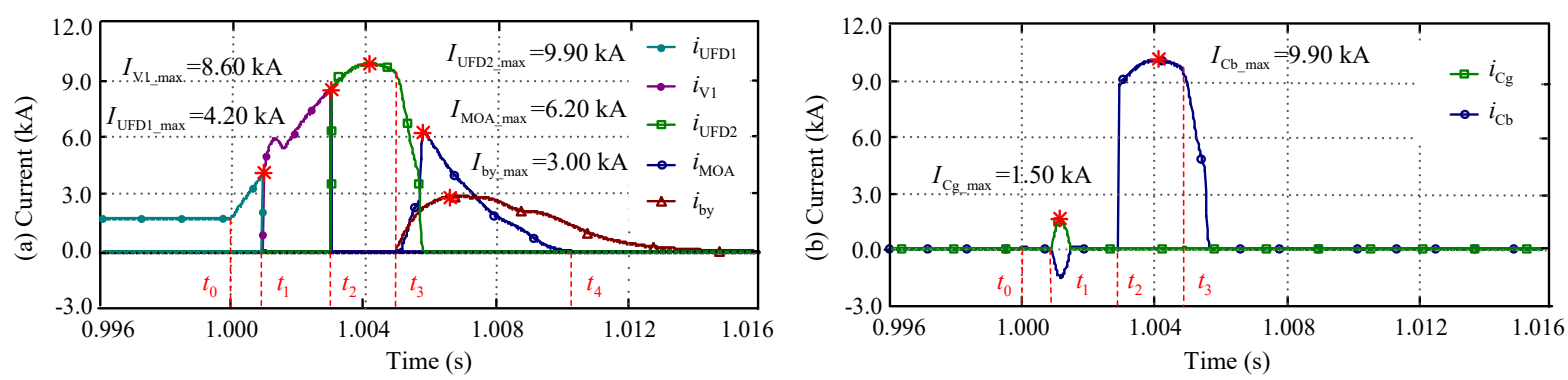

Figure 3. Fault isolation process. (a) Currents of each branch (b) Currents of $C_{\mathrm{b}}$ and $C_{\mathrm{g}}$.

\subsection{Reclosing identification process}

Assuming that the temporary fault has eliminated before $1.310 \mathrm{~s}$, the currents of reclosing identification branch and discharging branch are shown in Figure. 4 (a) and Figure.4(b), respectively. At $1.310 \mathrm{~s}, \mathrm{UFD}_{3}$, $\mathrm{UFD}_{4}$ will be turned on to drain the energy of $C_{\mathrm{b}}$ and identify the fault property. At $1.312 \mathrm{~s}$, the contactors of $\mathrm{UFD}_{3}$ and $\mathrm{UFD}_{4}$ have connected completely, and the $\mathrm{V}_{3}$ will be trigged at this moment. When the fault is a temporary fault, the fault point does not exist at 1.312s. Correspondingly, the maximum current of reclosing identification branch is $0.95 \mathrm{kA}$, which is less than the identification threshold value $I_{\text {th }}$ definitely. The discharging branch provides a discharging path for the $C_{\mathrm{b}}$, the maximum current of discharging branch is

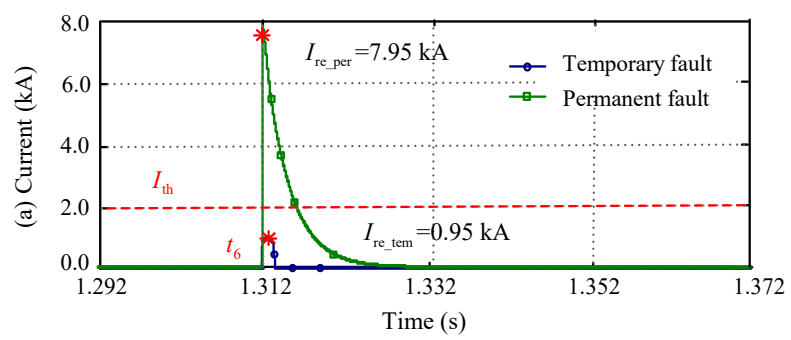

$2.95 \mathrm{kA}$. When the fault is a permanent fault, the fault point still exists at $1.312 \mathrm{~s}$, the maximum current of reclosing identification branch is $7.95 \mathrm{kA}$, which is larger than the identification threshold value $I_{\text {th }}$ definitely, so that the maximum current of the discharging branch is $0.05 \mathrm{kA}$, which is less than the one in the temporary fault. At $1.362 \mathrm{~s}$, UFD 5 will be turned on to drain the energy of $C_{\mathrm{g}}$. In summary, the fault type, either temporary or permanent, can be identified according to the current of reclosing identification branch. Correspondingly, the power electronic devices will not suffer from the large secondary overcurrent even under permanent fault condition. This is a core advantage of the proposed DCCB with adaptive reclosing strategy compared with the traditional method which recloses the DCCB directly.

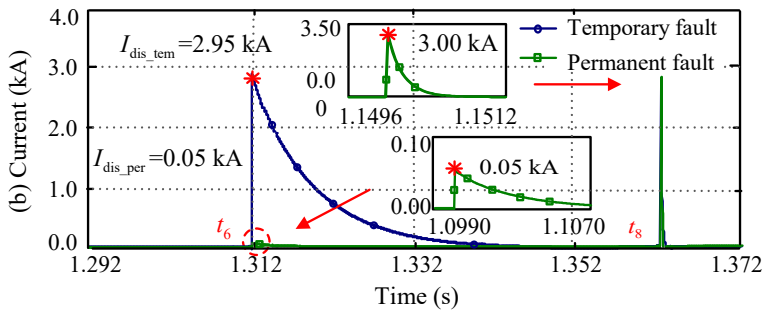

Figure 4. Reclosing identification process. (a) Currents of reclosing identification branch (b) Currents of discharging branch.

\section{Conclusion}

A novel current-commutation DC circuit breaker with adaptive reclosing capability is proposed in this paper.
The fault isolation and reclosing identification process of the proposed DCCB are analyzed, and the parameter of the devices is analyzed. The conclusions are as follows. 
1) Compared with the hybrid DCCB, the power electronic breaking branch composed of lots of IGBTs is replaced by the current- commutation branch, which is employed to interrupt bi-directional dc fault current.

3) In addition to providing a current flow path for the reclosing identification branch, the bypassing branch is employed to reduce the energy dissipation of MOA and accelerate the decay of the residual fault current.

3) Different from the auto-reclosing strategy, the proposed DCCB can identify the permanent fault and temporary fault by the current of the reclosing identification branch, which can avoid reclosing under permanent fault condition.

\section{Reference}

1. T. An, C. Han, Y. Wu et al., "HVDC grid test models for different application scenarios and load flow studies, " Journal of Modern Power Systems and Clean Energy, vol. 5, no. 2, pp. 262-274, Jul. 2017.

2. B. Li, H. Cui, F. Jing et al., "Current-limiting characteristics of saturated iron-core fault current limiters in VSC-HVDC systems based on electromagnetic energy conversion mechanism, "Journal of Modern Power Systems and Clean Energy, vol. 7, no. 2, pp. 412-421, Mar.2019.

3. L. Zhang, D. Zhang, T. Hua et al., "Reliability evaluation of modular multilevel converter based on Markov model, " International Journal of Electrical Power \& Energy Systems, vol. 7, no. 5, pp. 13551363, Sept.2019.

4. J. Xu, S. Zhu, C. Li et al., "The enhanced DC fault current calculation method of MMC-HVDC grid with fault current limiters, " IEEE Journal of Emerging and Selected Topics in Power Electronics, vol. 7, no. 3, pp. $1758-1767$, Sept. 2019.

5. G. Liu, F. Xu, Z. Xu, et al., "Assembly HVDC breaker for HVDC grids with modular multilevel converters," IEEE Trans. Power Electron, vol. 32, no. 2, pp. 931-941, Feb. 2017.

6. C. Li, C. Zhao, J. Xu, et al., "A Pole-to-Pole ShortCircuit Fault Current Calculation Method for DC Grids," IEEE Transactions on Power Systems, vol. 32, no. 6, pp. 4943-4953, Nov. 2017.

7. J. Xu, D. Wu, J. Li, et al., "The Voltage Clamping based DC Circuit Breaker with Decoupled Fault Isolation and Energy Dissipation Processes," IEEE Transactions on Power Delivery, doi: 10.1109/TPWRD.2020.2977055.

8. W. Shi, D. Cao, B. Yang, et al.,"500kV commutation-based hybrid HVDC circuit breaker, " Automation of Electric Power Systems, vol. 42, no. 7, pp. 102-107, 2018.

9. S. Tang, G. Jia, C. Zhang, et al., "Topology of precurrent-limiting DC circuit breakers for DC power grids", Automation of Power Systems, vol. 44, no. 11 , pp. 152-168, 2020 . 